\title{
El liderazgo escolar entendido desde el directivo y el profesorado en educación superior
}

\author{
Rubi S. Peniche Cetzal \\ Universidad Autónoma de Aguascalientes \\ rupeniche81@gmail.com \\ Cristóbal C. Ramón Mac \\ Universidad Autónoma de Aguascalientes \\ ccrmac@gmail.com \\ Victor M. Rosario Muñoz \\ Universidad de Guadalajara \\ vrosario@sems.udg.mx
}

Resumen: El propósito de este estudio fue determinar la opinión que tienen profesores $y$ directivos respecto del liderazgo escolar en instituciones de educación superior. El trabajo de campo se realizó en 3 IES ubicados en Yucatán, México; se recurrió a entrevista, grupos de enfoque y análisis de contenido. Participaron los 3 directores de cada centro, así como 21 profesores. Los directivos conciben a un líder escolar como una persona con mucha apertura a críticas, que no le incomode la opinión de los demás, y que permita recibir propuestas de mejora para la organización; como una persona bumana, con actitud de servicio para los demás. Las características son parecidas a las que los profesores desean de un líder, lo que parece ser una total paradoja, cuando los escenarios son opuestos.

Palabras clave: liderazgo escolar; profesor; directivo escolar; educación superior. 


\begin{abstract}
The purpose of this study was to determine the opinion of teachers and principals about school leadership in higher education institutions. The fieldwork was carried out at three schools located in Yucatan, Mexico, and data was collected by means of interviews, focus groups and content analyses. The directors of each of the three schools as well as 21 teachers participated in the study. The principals conceive of a school leader as a person who is open to criticism, who is not averse to the opinions of others, and who accepts proposals for improving the organization. They also see a leader as human, and motivated to be of service to others. The characteristics described by the principals are similar to those that the teachers want in a leader, which seems to be a total paradox, as the scenarios are the opposite.
\end{abstract}

Keywords: school leadership; teacher; school principal; higher education. 


\section{Introducción}

El estudio del liderazgo en las organizaciones aporta un sinfín de enfoques y reflexiones sobre este fenómeno; según algunos autores, el liderazgo es un tema ampliamente estudiado, pero continúa siendo el menos comprendido (Maureira, 2004). Su complejidad se hace evidente a nivel de la organización en general, sobre todo si se trata de una institución educativa, en donde el trabajo se basa esencialmente en las interacciones de distintos agentes educativos.

$\mathrm{Al}$ estudiar la función directiva para conocer las percepciones de directivos y las problemáticas que enfrentan en su desempeño cotidiano, Lorenzo (2005) menciona que se cuenta con directivos que ejercen un «liderazgo a la defensiva», lidiando con colegas desanimados, desinteresados y más preocupados por su condición personal o de pequeño grupo. En otro estudio sobre la función directiva, pero en secundarias públicas mexicanas, Aguilera (2008) señala que se encontró que en las escuelas donde el desempeño de los directores es eficaz, los procesos escolares y de aula tienden a serlo; de la misma manera, cuando los directores son ineficaces, las escuelas que dirigen también lo son. De manera particular, se observó que los directores de las escuelas eficaces incorporaban a los docentes y principalmente al equipo directivo en las decisiones escolares.

De acuerdo con Frost (2010), el liderazgo representa un factor esencial para las escuelas, pues permite ejercer una influencia que lleva al aprendizaje en diferentes niveles (alumnos, profesores, la escuela como organización y el sistema dentro del cual las escuelas operan), necesario para afrontar el desafío de la innovación. Reforzando esta idea, Lorenzo (2005) menciona que el liderazgo representa una propuesta de mejora incuestionable para la organización de las escuelas, siendo su estudio no solo imprescindible, sino ilusionante y utópico para los directivos y para todos los que comparten el mismo proyecto educativo del centro.

En la educación superior, el terreno para el estudio del liderazgo parece ser más complejo; las organizaciones escolares de nivel superior son ambientes multivariados, autónomos, dando lugar a escenarios particulares según los objetivos de formación y de ambiciones. La profesionalización de los directivos difícilmente ha sido valorada como una pieza clave para el logro de la calidad educativa. La propuesta realizada por la Asociación Nacional de Universidad e Instituciones de Educación Superior (ANUIES, 2006) en México permite situar al directivo en el entramado correspondiente a los retos de la educación superior. De esta manera, se presenta y se visualiza en la figura 1 una simbiosis de los diferentes desafíos. 


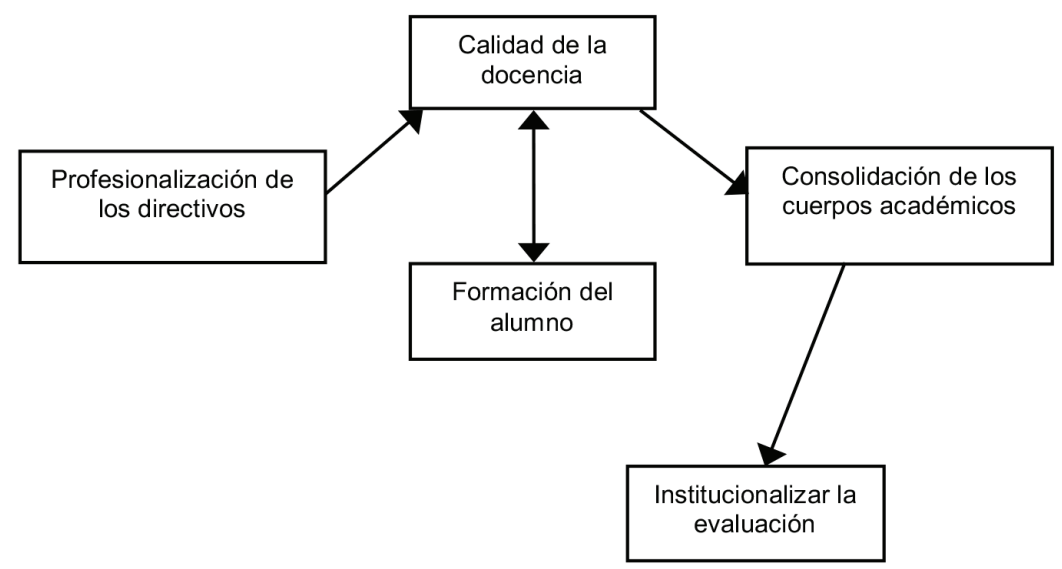

Figura 1. Simbiosis de los desafíos y retos de la educación superior según la ANUIES.

Este esquema permite observar cinco retos que las universidades e instituciones de educación superior deber enfrentar, siendo el primer desafío la profesionalización de los directivos. Como ya se ha mencionado, el directivo se mantiene como la máxima autoridad en una organización y a través del cumplimiento de sus funciones permite generar el eficaz desarrollo de la misma, por lo que se encuentra fundamentada su ubicación como el primer reto que corresponde atender.

Ante lo anterior, surge el interés por indagar qué es lo que opinan los profesores y directivos de lo que debe ser un líder escolar, considerando la comparación de estas opiniones. Para ellos, este estudio se rigió por los siguientes objetivos.

Objetivos de investigación

1. Describir la opinión de los profesores de educación superior respecto del papel de un líder educativo.

2. Describir la opinión del propio directivo escolar de una institución de educación superior respecto del papel que juega un líder.

3. Comparar la opinión que tienen los profesores y directivos respecto de lo que es un líder escolar.

\section{El liderazgo como eje transversal en el gobierno del centro escolar}

El liderazgo es un indicador fundamental en la eficacia de los centros educativos, pues existe suficiente bibliografía pedagógica con ejemplos positivos de escuelas que han logrado obtener niveles elevados de calidad gracias a un liderazgo eficaz 
del director y a las adecuadas expectativas de los demás agentes educativos (Maureira, 2004). Es por ello que ante estas afirmaciones el liderazgo forma parte del conjunto de términos básicos de los movimientos de escuelas eficaces, mejora escolar y cambio educativo (Fullan, citado por Lorenzo, 2005); además, forma parte de la llamada Gestión de Calidad, del Modelo Europeo y de la corriente integrada de la Mejora para la Gestión de Calidad.

Estos movimientos mencionados por Lorenzo (2005) y el interés en el liderazgo educativo se derivan de la necesidad de producir cambios significativos en el funcionamiento de los sistemas educativos y, de manera más particular, en los centros escolares. Ante esta situación, como señala Gago (2008), se considera importante que el papel de agente de cambio forme parte del perfil directivo, solamente que esto no será suficiente, pues, de igual manera, el reto radica en activar las energías y capacidades del profesorado.

Continuando con la vinculación entre el liderazgo de los directivos y los efectos en la calidad de los centros educativos, conviene hacer mención de la atractiva propuesta que Cantón y Arias (2008) realizan como resultado de los estudios que han efectuado en diversas escuelas de España; señalan que para lograr una dirección de calidad en los centros educativos es necesario abogar por el liderazgo de la dirección que se concrete en el estilo democrático, participativo y descentralizador en la toma de decisiones, posibilitador, animador y comprometido con el cambio. Además, como bien puntualiza Anderson (2010), los líderes educativos influyen en el mejoramiento escolar, sobre todo si se comprometen y realizan prácticas que afecten positivamente a las motivaciones de los maestros, sus habilidades y capacidades profesionales, y las condiciones de trabajo en que se desempeñan. Como consecuencia, continúa Anderson, la modificación de las prácticas docentes impactará en los resultados de los estudiantes.

A este mismo tenor, el liderazgo es un desafío en la vida de las personas y de las organizaciones, y que, independientemente del medio, constituye el factor determinante para el logro de los objetivos deseados (Rubio, 2006). Es por ello que surge la necesidad imperiosa de generar los mecanismos idóneos para formar líderes eficaces en todos los niveles organizacionales, de manera que en conjunto se realicen cambios favorables para el crecimiento y desarrollo de la institución.

Al ser el eje transversal de las organizaciones y de los centros educativos, el liderazgo adquiere una gran importancia para las investigaciones y estudios vinculados con la eficacia de las escuelas y de quienes ocupan la dirección. Para ello, es necesaria la delimitación de competencias o de un perfil en el que se establezcan las características y funciones que los líderes deben cubrir para que las instituciones maniobren según sus prioridades y con base en la planeación correspondiente. 


\section{Competencias del liderazgo en el ejercicio de la dirección}

Ante el debate de quién debe ser líder, resulta apremiante tener claro que ejercer el liderazgo en un centro educativo no es necesariamente dirigirlo, ya que es posible ser el director y no ser el líder. En contraste, se puede ser un simple profesor o tutor y ejercer entre los compañeros o alumnos una importante función de liderazgo (Lorenzo, 2005). Sin embargo, es preciso que sean los directores quienes ejerzan un liderazgo adecuado para que, aunado al buen desempeño de sus funciones administrativas, sea posible la mejora del centro educativo.

En México, el director ha comprobado esa ruptura entre el desarrollo de sus funciones como líder de la institución y la presión administrativo-burocrática a la que es sometido por parte de las Administraciones públicas, siendo esta última la que le consume una mayor parte de su tiempo (González, Silva, Antúnez y Carnicero, 2008); situación que desencadena inconformidad en los directivos, quienes se encuentran en el dilema de formarse para ser líderes educativos o para desempeñarse de manera acorde a las funciones que el mismo sistema educativo les exige. De acuerdo con Martínez (2008), existen ciertas habilidades que los directivos deben desarrollar para enfrentar el dilema entre lo meramente administrativo y la coordinación de esfuerzos como un verdadero líder: comprensión y análisis de las políticas educativas, del contexto, normatividad, métodos, evaluación; visión holista de la institución; y sensibilidad para relacionarse con otros.

$\mathrm{Al}$ respecto, Trujillo (2007) señala que el director de un centro educativo debe promover, en vías de la ejecución de su liderazgo, un idealismo compartido y una visión generada en conjunto, con la finalidad de que todos se identifiquen con los fines y hagan suyos los propósitos de la institución. García (2008) hace referencia a las estrategias que Blase y Blase (2002) señalan como aquellas que emplean los directores en su papel como líderes de la educación, las cuales son: sugerencias, retroalimentación, formación, cuestionamiento, solicitud de consejo y opiniones y la visibilidad; esta última requiere de altos niveles de conocimiento profesional, estrategias y comprensión.

El líder y el directivo son dos personajes que realizan la misma función en dos tipos diferentes de grupo, el primero, en los espontáneos, y el otro, en los organizados. A esta idea corresponde la utilización relativamente frecuente del término «líder formal» aplicado al directivo y la de «líder informal» para aquel que no es directivo (Uriz, 1994: 199). Pese a lo anterior, existe un mayor énfasis en la concepción de los líderes como administrativos, pues al tener la autoridad, solamente requieren de poder e influencia para llevar a cabo las transformaciones 
organizacionales que se requieren. De ahí que Henry Mintzberg haya identificado diez funciones que los líderes realizan para lograr los objetivos. Para una mejor comprensión, se agrupan las funciones en tres categorías: interpersonales, informáticas y decisionales (Lussier y Achua, 2002).

Las diez funciones del líder variarán según el tipo de organización y de los recursos con los que cuente. Asimismo, existe la posibilidad de que diferentes personas lleven a cabo funciones distintas, pero con la firme intención de complementar los esfuerzos para el logro de los objetivos; y es poco probable que un mismo líder desempeñe todas las actividades que las funciones administrativas exigen. Con esta idea coincide Bush (2016), quien incluso menciona que esta situación debe ser abordada en la formación de líderes educativos, pues el patrón de formación adoptado por cada nación refleja lo que se considera apropiado para apuntalar la calidad de la educación en el siglo xxi, y por ello es de vital importancia reconocer la cultura y el contexto específicos si lo que se busca es proveer una educación de la mayor calidad posible.

En el caso particular de las organizaciones o instituciones educativas, estas funciones encajan en el puesto directivo, siendo esporádica la intervención de los profesores para el ejercicio de las mismas, pues, como señala Trujillo (2007: 4), «el director se constituye como el eje direccional, para lo bueno y para lo malo, es el punto de fijación de todas las miradas», teniendo como responsabilidad básica el conocimiento y consideración de las expectativas y necesidades de los profesores, del alumnado y de los agentes sociales del entorno, incluyéndose en este ámbito los padres de familia, dependiendo del nivel educativo de que se trate, pues es sabido que a partir de la educación media superior es mínima la participación de los padres en la formación de sus hijos.

Cabe resaltar que Cáceres (2007) señala que la variable ambiental ejerce un papel decisivo en el desempeño de estas funciones, de tal forma que el director deberá encarnar un determinado rol o modelo directivo según el estilo organizativo presente en la institución, esto es, contextualizando su práctica gestora y directiva a las características concretas del centro en el que se sitúe.

Fernández, Álvarez y Herrero (2002) señalan que ante los cambios vertiginosos del entorno los líderes deberán desarrollar las competencias fundamentales que permitan a la organización continuar con su desarrollo y crecimiento, siendo estas las que a continuación se plantean:

a. Lectura inteligente de la realidad e intuición de perspectivas de futuro.

b. Niveles altos de autoestima y fuerza emocional para manejar el cambio y las consecuencias. 
c. Agilidad para diagnosticar permanentemente los puntos fuertes y áreas de mejora de la empresa.

d. Capacidad para implicar a sus colaboradores en el [sic] política y estrategia de la institución.

e. Voluntad para compartir el liderazgo. El líder del futuro tendrá que dominar el arte de la comunicación y del trabajo en equipo.

Esta última competencia da cuenta de la imperiosa necesidad de contar con un liderazgo compartido, en el que se involucren los distintos agentes que conforman la comunidad educativa. Asimismo, contar con habilidades para la planeación estratégica constituye un elemento imprescindible para el adecuado funcionamiento de la organización. Para esto, es necesaria la capacidad de trabajar en equipo y poseer las actitudes que la favorezcan. Una competencia interesante que ha sido considerada como parte de esta lista es la inteligencia emocional, elemento frecuentemente olvidado para el desempeño de todo líder, pero útil para el manejo de las emociones y el estado anímico para la interacción con los demás.

Johnson y Uline (2005) señalan que el Interstate School Leadership Licensure Consortium (ISLLC) desarrolló un conjunto de estándares para informar sobre la preparación y continuo desarrollo profesional de los administradores escolares. Estos estándares publicados en 1996 se vinculan con el liderazgo y trascienden en la Administración educativa, pues son útiles para los dirigentes y para la formación de nuevos líderes en la organización. Los estándares han sido agrupados en seis grandes áreas de conocimiento, disposiciones y funciones, incluyendo el de los seguidores (figura 2).

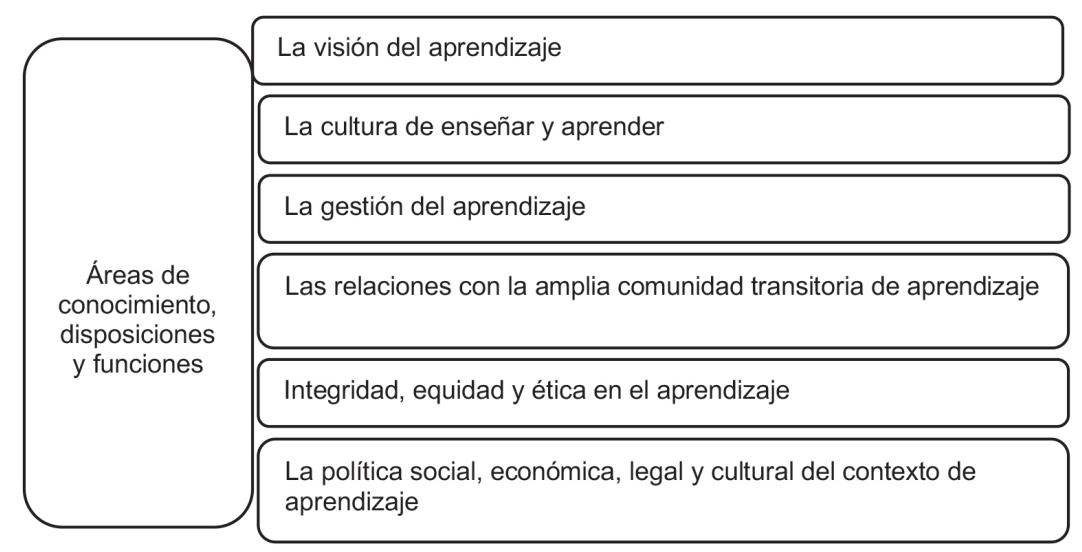

Figura 2. Áreas de preparación y desarrollo profesional de los administradores escolares (Johnson y Uline, 2005). 
Una propuesta más reciente la representan los estándares profesionales para líderes educativos, emitidos por el National Policy Board for Educational Administration (2015), que consiste en un conjunto de ocho estándares con los que se procura asegurar que los líderes educativos estén preparados para enfrentar de manera efectiva los desafíos y oportunidades del trabajo diario y futuro, tomando en cuenta que la educación, las escuelas y la sociedad están en continua transformación. Los estándares propuestos son: 1) misión, visión y valores fundamentales; 2) ética y normas profesionales; 3) equidad y responsabilidad cultural; 4) currículo, instrucción y evaluación; 5) comunidad de cuidado y apoyo para estudiantes; 6) capacidad profesional del personal escolar; 7) comunidad profesional de maestros y personal; 8) involucramiento significativo de las familias y la comunidad; 9) operaciones y administración; y 10) mejora escolar.

Los dos grupos de estándares representan un avance en la integración de áreas y funciones que deben ser propias de los directivos. Asimismo, la riqueza de esta aportación no solamente radica en la especificación del quehacer del líder, sino que considera la complementariedad de sus funciones con aquellas que son propias de los seguidores.

Con respecto a la situación de las universidades e instituciones de educación superior, la ANUIES en México señala que uno de los retos que deberá enfrentar la educación es la profesionalización de los directivos, quienes son agentes importantes para generar un sistema innovador y con calidad. En la visión que se tiene para 2020 se establece que los roles de profesores, directivos y alumnos deben ser redefinidos, de manera que los profesores sean tutores y facilitadores del aprendizaje; los alumnos, más activos y responsables en su proceso formativo; y los directivos, más académicos y profesionales (Tolentino, 2007).

Asimismo, Martínez Rizo (2000) hace referencia sobre lo fundamental que es el liderazgo directivo en las organizaciones educativas, agregando que es necesario contar con las cualidades que le permitan cumplir con su función. Algunas de ellas son: a) experiencia y conocimiento del campo especializado en que se ubica el quehacer institucional; b) capacidad organizativa y de gestión; c) habilidades para conjuntar esfuerzos y dirigir grupos de trabajo; y d) visión de futuro y capacidad de transmitirlas. La propuesta de Martínez (2000) se resume a través de las siguientes características que la ANUIES plantea en cuanto al personal directivo de las IES:

a. Personas con preparación académica y experiencia académico-administrativa.

b. Fuerte liderazgo académico en el interior de sus comunidades y en su entorno social; promotores de innovaciones. 
c. Capacidad para definir la misión y visión institucional, así como la planeación, la evaluación y la toma de decisiones; visión amplia de las problemáticas en la educación superior; dominio de técnicas de dirección y gestión.

d. Promotor de la superación constante de su personal.

e. Desempeño eficaz y eficiente apoyándose oportunamente en las tecnologías de la información.

Como es visto, para las instituciones de educación superior (IES) existe una serie de indicadores o parámetros que determinan el papel de un dirigente escolar, sin que esto signifique un manual a seguir.

\section{Metodología del estudio}

El estudio aquí reportado fue de corte cualitativo y se llevó a cabo utilizando un diseño de estudios de casos, tipo colectivo (Stake, 2010), siendo empleado este cuando el interés del investigador se centra en una población, un fenómeno o condición general, seleccionando varios casos que se han de estudiar. Se trabajó con tres universidades públicas del estado de Yucatán, México, las cuales se caracterizaron por ser las más grandes en su tipo, en la región. Los actores fueron los directivos escolares ( 3 rectores) y profesores de cada universidad ( 21 profesores); el trabajo de campo se realizó durante 6 meses (período enero-junio 2015). La obtención de los datos fue a través de entrevistas semiestructuradas, análisis de documentos, observaciones (reuniones con personal directivo, reuniones con personal docente).

\subsection{Análisis de datos}

Todas las entrevistas, tanto personales como del grupo focal, fueron audiograbadas. Para las transcripciones de las entrevistas de profesores y del director, así como del grupo focal, se optó por respetar las convenciones ortográficas según Farías y Montero (2005). Como parte del análisis y para un mejor entendimiento del proceso, se identificaron las intervenciones del investigador y del entrevistado, anteponiendo las letras $\mathrm{P}$ (profesor) y D (director). Para el análisis de la información, se prosiguió a elaborar un conjunto de esquematizaciones (como bien muestra la tabla 1) que permitieran seleccionar aquellos fragmentos de la información, con el objetivo de ir entendiendo razones para la interpretación de los datos remarcados en las entrevistas; entendiendo esquematización como la estrategia gráfica que permite organizar y segmentar la información para conseguir un entendimiento y significado de los datos. 
En la siguiente tabla, se muestra un ejemplo del análisis de contenido en la entrevista realizada a un profesor.

Tabla 1. Ejemplo del análisis en una tabla discursiva

\begin{tabular}{|c|c|c|c|}
\hline I, P o D & Unidad de análisis & Codificación & Categoría \\
\hline $\begin{array}{c}\mathrm{P} \\
\text { (profesor) }\end{array}$ & $\begin{array}{c}\text { «.. hay compañeros que se siente } \\
\text { más fuertes si son amigos del } \\
\text { director...» }\end{array}$ & $\begin{array}{c}\text { P-D } \\
\text { (entendiendo poder desde la } \\
\text { visión docente) }\end{array}$ & Poder \\
\hline
\end{tabular}

Con respecto del reporte de observación, fue necesaria la elaboración de una tabla que contempló aspectos relativos a momentos llamativos del evento, tipo de personaje expresado e identificado, comentarios alternativos, comportamientos llamativos y resultados alcanzados en el evento. Al final, el investigador procedió a colocar una etiqueta que pudiera acoplar el fenómeno identificado en el apartado de la tabla.

Tabla 2. Ejemplo de análisis de la observación de una reunión de profesores

\begin{tabular}{|c|c|c|c|c|c|}
\hline Momento & Personaje & Comentario & Comportamiento & Efecto & Etiqueta \\
\hline \multirow{4}{*}{ Intermedio } & $\begin{array}{c}\text { Profesor } \\
\text { (hombre, } \\
\text { joven) }\end{array}$ & $\begin{array}{c}\text { mi tiempo en } \\
\text { estas cosas. ¿Ya } \\
\text { viste el horario? } \\
\text { Está pésimo». }\end{array}$ & $\begin{array}{c}\text { Indiferencia } \\
\text { Muecas }\end{array}$ & $\begin{array}{c}\text { Distracción } \\
\text { en otros }\end{array}$ & Participación \\
\hline
\end{tabular}

En el análisis formal de documentos, la manera por la cual se logró un análisis más puntual de la información contenida fue a través del empleo de una tabla donde convergieron los tres recursos de contenido, y donde se describen elementos que pudieron caracterizar los datos hallados en cada uno. A continuación, se muestra una aproximación del análisis que se realizó, siendo necesaria la integración de los tres recursos.

Tabla 3. Ejemplo del análisis de contenido de los datos obtenidos

\begin{tabular}{|c|c|c|c|c|}
\hline Datos & Tipo & Propósito & Unidad & Categoría \\
\hline Himno & Texto, ritmo & $\begin{array}{c}\text { Despertar el } \\
\text { espíritu }\end{array}$ & $\begin{array}{c}\text { Valores } \\
\text { Propósitos del centro }\end{array}$ & Ética \\
\hline Escudo & $\begin{array}{c}\text { Ilustrativo, } \\
\text { emblema }\end{array}$ & $\begin{array}{c}\text { Inspirar } \\
\text { identidad }\end{array}$ & $\begin{array}{c}\text { Identidad } \\
\text { institucional }\end{array}$ & Compromiso \\
\hline $\begin{array}{c}\text { Plan } \\
\text { estratégico }\end{array}$ & Texto, metódico & $\begin{array}{c}\text { Determinar } \\
\text { visión compartida }\end{array}$ & Metas compartidas & $\begin{array}{c}\text { Trabajo } \\
\text { profesional }\end{array}$ \\
\hline
\end{tabular}




\subsection{Validez}

Para fines del estudio, se llevó a cabo cierta estrategia metodológica hacia la validez de los resultados: una triangulación, siendo esta un esfuerzo por ver si aquello que observamos y de lo que informamos contiene el mismo significado cuando lo encontramos en otras circunstancias (Stake, 2010). Específicamente se trabajó sobre una triangulación metodológica (Denzin, 1989). Otra propuesta que manifiesta Stake (2010) y que complementa este trabajo de triangulación fue la llamada revisión de los interesados, y donde se le pide al actor que examine escritos en borrador o grabaciones, en los que reflejan actuaciones o palabras suyas, se le pide que revise la exactitud y la adecuación del material.

\section{Resultados}

Considerando los objetivos del estudio, los hallazgos son presentados en función de lo que los profesores determinaron respecto del liderazgo en un primer apartado, y posteriormente, lo que los directivos manifestaron al respecto. Los resultados fueron obtenidos considerando tanto las entrevistas como las observaciones hechas en los centros y del análisis de documentos obtenidos.

\subsection{Entendimiento del liderazgo desde la óptica docente}

Los docentes entrevistados manifestaron una serie de comentarios respecto de su entender del liderazgo en la institución. Figuraron situaciones de gran interés, entre las cuales se muestra que estos profesores consideran que el liderazgo debe ser parte inherente del trabajo de cualquier profesional de la educación, y más del directivo del centro.

En primer lugar, se describe lo que estos docentes consideran que todo líder educativo debe poseer sin necesariamente tener un puesto directivo.

$\mathrm{P}:$ «[...] un docente líder, reflexivo, pensante, que tenga amplios contenidos de la realidad que se está viviendo, y tenga enfoque y oriente al alumno al qué hacer pero de la vida actual y no se quede en el pasado, que sea futurista [...] buscar todas esas estrategias, de armarlas, dárselas y después estar calificando, hacer una evaluación sumativa al final y darles una calificación pero que en verdad se hayan desarrollado muchas habilidades y destrezas a través de las tareas y es un docente que diariamente tiene que estar calificando, revisando y evaluando, actualizado totalmente tomando cursos para que ya pueda estar a distancia de sus alumnos $[\ldots] »$. 
Los profesores coinciden en describir a un líder educativo como todo aquel sujeto que procura el desarrollo integral del estudiante, y más en jóvenes universitarios; también se puntualiza la necesidad de que todo profesional de la educación promueva en los alumnos un espíritu humano y visionario, con ganas de triunfar y superarse. Con base en lo identificado en algunas observaciones, los directivos promueven una constante actualización en beneficio del desempeño profesional de los docentes; de igual manera se fomenta la creación de espacios para la participación de los jóvenes en sus diferentes actividades o proyectos.

Parece que un líder educativo debe firmemente buscar y lograr un cambio en los estudiantes universitarios, siendo estos el futuro de la sociedad. Se comenta que los estudiantes siempre buscan oportunidades de crecimiento, y que eso depende de los alientos que puedan obtener de sus formadores en su educación superior.

Con lo anterior, se pone de manifiesto que para los profesores el liderazgo no está necesariamente ligado a un puesto directivo, y que para que las instituciones de educación superior puedan lograr el impacto esperado para el país, estas organizaciones deberían estar conformadas por líderes que deseen transformar la vida de las personas que pasan ante ellos en las aulas. Que no se vea solamente el trabajo profesional como un pretexto para enriquecerse de halagos, niveles laborales, o ingresos económicos cada vez mayores.

$\mathrm{P}:$ «[... $]$ para que seas líder tienes que lograr diferencias en tu camino, no solo te intereses en ti, no ser egoísta; el ser un profesor universitario es una gran responsabilidad que la sociedad te encomienda».

$\mathrm{P}:$ «[... $]$ no solo se debe pensar en producir, tienes que pensar en los que esperar de ti. Los muchachos son nobles, y esperarían lo mejor de cada uno».

Es notable e interesante que los mismos profesores esperarían que ellos pudiesen servir como agente de cambio, sabiendo que no es fácil pero que con el compromiso diario y un espíritu humano podrían dar un giro a la educación superior, con la esperanza de formar también a líderes que cambien la sociedad.

Respecto del liderazgo de un directivo escolar, se identificó lo siguiente.

$\mathrm{P}: \ll[\ldots]$ todo directivo debe planear, debe ser también con una política de comunicación muy abierta, haya transparencia en su parte administrativa, que dé confianza y delegue a todos sus subdirectores, jefes de departamento, que brinde confianzas también a su gente, que reconozca y valore el trabajo de todos su equipo».

Se puede entender que el directivo se mantiene enfocado más al grupo de trabajo que lo rodea, descuidando a los demás que no se encuentran es ese círculo organizacional. Los entrevistados manifiestan abiertamente este sentir relativo a 
que su directivo no puede disimular su inclinación o preferencia por los que le sirven o le trabajan de cerca. Para ellos, existe cierta incomodidad cuando el director tiende a convivir con sus subdirectores de área; en algunas reuniones informales el director difícilmente se aproxima con los profesores o demás empleados del centro. Pudo notarse este dato durante una reunión de trabajo observada, y donde el directivo escolar mantenía cercanía e intercambio de información con un grupo de profesores muy específicos; en otro caso, con una situación similar, se logró escuchar entre algunos profesores comentarios relativos a «maestros preferidos».

En algunos casos, los directivos sí se muestran accesibles, con algunos gestos de amabilidad, pero no son suficientes cuando podrían tener un mejor acercamiento con los demás que no sean de su equipo directivo.

$\mathrm{P}:$ «[...] si es bueno, pero lo que no me agrada es que se la pasa encerrado en su oficina y siempre es con su grupo de trabajo».

$\mathrm{P}: «[\ldots]$ casi nunca lo vemos, casi no se nos acerca».

$\mathrm{P}:$ «[... ] solo está con su grupo de trabajo. A veces sentimos que nos saluda por compromiso».

$\mathrm{P}:$ «[... $]$ yo creo que si director se abriría más a nosotros, podríamos trabajar más en confianza».

Con esto se denota, la urgencia que manifiestan los profesores respecto a la cercanía del director hacia ellos; parece ser una necesidad diaria.

$\mathrm{P}:$ «[...] que tome en cuenta a la gente, se basa en su pequeño grupo de trabajo [...] se reduce el impacto en las decisiones que toman y eso hace de que muchos maestros sean como indiferentes, que no tomen en cuenta ni su trabajo. Debería ser sencillo».

$\mathrm{Al}$ parecer, los profesores manifiestan que todo se traduce en la falta de humildad del director, siendo este uno de los principales valores que debería estar presente para que todo individuo que dirige a otros pueda lograr la confianza y, así, caminar sin problema hacia cualquier objetivo planteado. La humildad se comprende como el comportamiento íntegro del individuo sin considerar su posición jerárquica para tratar a los demás con mayor o menor atención; también un directivo humilde es aquel que no espera una ocasión especial para interactuar con los demás, sin que esta atención sea vista como fingida o comprometida porque otros lo observen.

Vale la pena comentar la diferencia que se encontró en dos directivos, haciendo referencia a la lista de asistencia que entregan al convocar a profesores a reuniones de trabajo: un directivo presentaba una lista formal con una frase mo- 
tivadora en la parte final de la lista, y el otro, solamente una lista de nombres. En el primer caso, el ambiente de trabajo se mostró más amable y cordial.

Es interesante saber que los docentes esperan a un director humilde, esperando verlo en cualquier pasillo del centro escolar platicando con cualquier estudiante o empleado, sin problema alguno, y que esta plática no sea necesariamente de asuntos escolares o laborales.

$\mathrm{P}:$ «[... $]$ para que veas al director fuera de su oficina está complicado. Solo lo vemos en reuniones obligatorias, en eventos oficiales de la escuela, ah, y en esos momentos está en el presídium».

$\mathrm{P}$ : «Pues si fuera más accesible sería otra cosa. Estoy segura de que sí se podría, pero algo les pasa cuando se convierten en jefes, se olvidan de que empezaron como nosotros».

Analizando los comentarios emitidos por los entrevistados, aparece otro factor de gran importancia: el compromiso que el director debería tener. Parece que el director se muestra inconforme cuando lo planeado no sale como desearía, pero lamentablemente las acciones son llevadas a cabo por indicaciones que el mismo director toma, lo que hace pensar que este no se incluye en los procesos ocurridos en la organización.

$\mathrm{P}:$ «[... $]$ sabemos que no somos perfectos, que a veces cometemos errores, que no entregamos nuestros documentos que nos piden, que no nos apuramos con otras muchas cosas, por tanta presión de trabajo, pero el director se muestra como el único ofendido».

Los profesores reconocen que comenten algunas faltas en sus labores, que en algunas ocasiones a pesar de las indicaciones o decisiones que hayan sido tomadas por el área directiva, ellos se equivocan, pero a pesar de esas situaciones y reconocer estos errores, el directivo se muestra totalmente ajeno a la escuela, y se comporta como un agente externo que solo se dedica a juzgar sin recordar que es parte de la organización.

Estos docentes esperarían un nivel alto de compromiso por parte del director, siendo reflejado en un acompañamiento de cualquier proceso masivo que se necesite realizar, hablando de procesos de acreditación, certificación o los tan cansados y necesarios programas de fortalecimiento institucional, considerando que los mismos profesores los describen de esa manera. En concreto, la institución desearía que el director escolar fuese un individuo inclusivo e involucrado en el centro, representando un pilar para atender dificultades presentadas. El compromiso de un director con su institución y en lo que acontezca en ella es un valor que puede ser aplaudido y admirado por todo equipo de trabajo. 


\subsection{Entendimiento del liderazgo desde la óptica del directivo.}

Después de analizar lo que los profesores consideran del liderazgo hacia su mismo trabajo y el de un directivo, ahora se analiza lo que este último comenta respecto a sus cualidades como líder y lo que le faltaría tener para ser un icono en la organización.

$D:$ «[...] yo trato de que en mi personal directivo, por ejemplo, haya representantes de todos los grupos, para que de esa manera ellos mismos sean los que peleen por los intereses de los demás, que yo tenga la información [...] vamos a traerlo, vamos a platicarlo aquí, vamos a ver cuáles son las decisiones de la institución que vamos a desarrollar».

Es clara la postura del directivo al manifestar que la suspicacia o la habilidad para determinar grupos contrarios a la administración es de suma importancia, pues permite anticiparse a situaciones que retrasen o impidan procesos necesarios de realizar. Estos directivos comentan que el observar siempre a su personal, ver el comportamiento de los demás, conocer sus gustos y preferencias, así como prestar atención a sus comentarios, permiten identificar esos posibles grupos de choque. $\mathrm{Al}$ respecto, pareció interesante durante una observación de reunión de trabajo de profesores con el propio directivo que mientras un coordinador académico daba instrucciones el directivo observaba a los profesores con detenimiento y tomaba nota; al tomar la palabra el funcionario, tomaba en cuenta lo que tenía en sus anotaciones para argumentar su discurso; fue notorio que mucho de lo comentado por él se basó en lo que los participante decían durante la sesión.

Parece sumamente interesante lo planteado al referirse a la necesidad de ubicar a sus profesores como problemáticos, para que de esta manera se decida cómo trabajar con ellos, o antes de que puedan crear alguna dificultad, se les otorgan tareas o actividades que los mantengan ocupados.

$\mathrm{D}:$ «[...] hay gente que tiene mucha capacidad, hay docentes que tienen mucha capacidad, tengo doctores acá, pero están dedicados a su propio rollo, el asunto es que son algo problemáticos; son personajes fuentes, comúnmente contrarios a lo que dicte la dirección».

D: «Lo importante es encontrar maneras de cómo disminuir su efecto sobre la administración, yo creo que hay que ocuparlos».

Estos comentarios pueden convertirse en un elemento de gran relevancia para comprender ciertos comportamientos que ocurren en una institución de educación superior. Se está entendiendo que para que ciertos integrantes del centro escolar no se conviertan en obstáculos a futuro, primero se deben identificar y 
proceder a conocer cada paso que dan. El director sabe muy bien las actividades que cada profesor o integrante de la escuela tiene que cumplir, y es de esta manera en que él puede anticiparse a lo que posiblemente pudiera obstaculizar lo que él consideraría como necesario para la institución.

Entonces, si el director sabe que cierto grupo de empleados, específicamente profesores, pudieran ser bandos contrarios o renuentes a contribuir en favor del centro, es necesario mantenerlos ocupados en tareas que demande más tiempo de su desempeño.

$\mathrm{D}: «[\ldots]$ cuando se trata de ese tipo de personas, trato de decir, ¿sabes? Vamos a meterlo al CONACYT, vamos a meterlo al [...] porque no le hablas a fulano de tal para que puedan hacer un trabajo conjunto, de mayor impacto $[. .$.$] y ya$ les damos sus viáticos para que puedan ir a ver a la otra persona, $y$ aparte nos traen un proyecto».

Parece asombroso cómo con este tipo de estrategias, traducidas en atenciones o beneficios otorgados a ciertos profesores, permitiría espacios para que la dirección se reste profesores problemáticos, y además, se obtenga de paso un beneficio que repercuta a la organización.

Si se considera esta medida como de poco juicio, es simplemente parte del actuar micropolítico en un centro de trabajo, sobre todo de un directivo que se mantiene con la idea firme de alcanzar sus objetivos. Circunstancias explicadas por Foucault (1991), al señalar que en la base de las luchas de poder se encuentran aquellas estrategias, visiones o intereses que pueden ofrecer más dependiendo del contexto, confirmándose que como acción «el poder es necesario para limitar al poder» (Flyvbjerg, 2001, p. 134).

En la siguiente figura se esquematiza el proceso que sigue el director escolar para evitar sujetos potencialmente conflictivos.

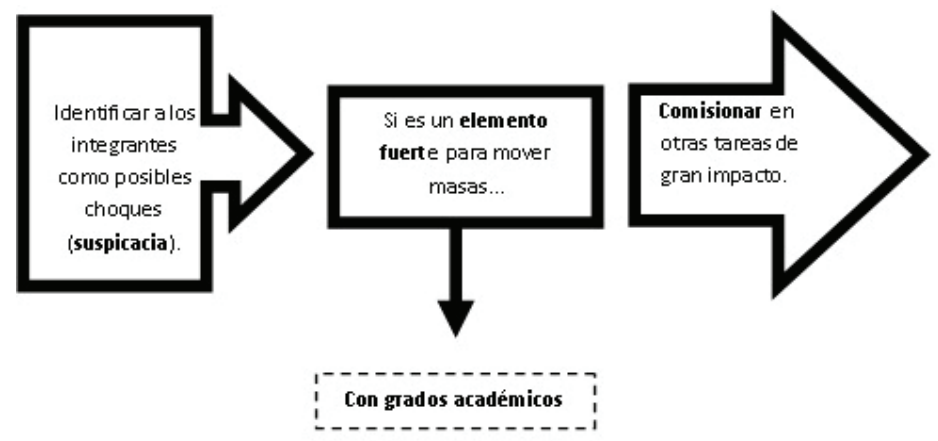

Figura 3. Estrategia directiva para evitar actores conflictivos. 
Quizá el director pretenda que aquellos profesores que se dedican a tareas de investigación o que tienen una preparación de alto nivel, llámense doctores o investigadores, puedan ser aquellos que pudieran influir sobre otros para actuar en contra o quizá solo ser jueces ante lo que la parte directiva establezca como mejoras para el centro escolar. Entonces, si eso se cumpliera parece que sería conveniente mantener alejados a esos supuestos profesores eméritos a través de espacios que puedan ser de interés tanto para los involucrados como para la escuela. Ante esto, Blase y Blase (2002) expresan que todo directivo organizacional recurre a medidas que le permitan conllevar su trabajo diario, y que en muchas ocasiones esas medidas son la menos esperadas o deseadas.

Continuando con esta caracterización del directivo escolar, la apertura que pueda demostrar este personaje es de suma relevancia para obtener la cercanía o confianza de los involucrados. Esto es lo que el entrevistado manifiesta poseer y se convierte en su mayor ofrecimiento para sus subalternos.

$\mathrm{D}:$ «Si vienen algunos maestros por ejemplo que tienen alguna idea muy específica y me doy cuenta de que está dentro de esos trabajos que se están desarrollando, les digo: ¡haz tu proyecto!, puedes hacer esto, aquellos, y así y así $y$ así. $Y$ se van felices y lo hacen. Yo he visto que cuando se le da apertura a la gente, se sienten satisfechos consigo mismos y cuando les vas a pedir algún apoyo, te dicen que sí te voy a apoyar porque sé que tú también ya me apoyaste $[\ldots]$ eso es como integrar $[\ldots] \gg$.

El profesor podría sentirse útil al obtener cierta accesibilidad de su directivo, que las puertas del área administrativa no se caractericen como inaccesibles. Esta apertura es remarcada por el director al comentar que sin ella los demás no podrían manifestar sus ideas, que, en la mayoría de los casos, son sorprendentes.

Asimismo, y en conjunto con esta apertura necesaria, se adjunta el humanismo que puede ser un factor que cualquier elemento organizacional reconocería positivamente en su autoridad, a pesar de las dificultades que se presenten en el centro. Si un directivo es humano, es decir, procura un necesario y oportuno entendimiento de los demás, que no sobrepase los derechos existentes, y trata con amabilidad y empatía a los otros, eso puede hacer la diferencia entre un ambiente crítico y otro que pueda involucrar a los miembros de la organización.

A pesar de que la cantidad de trabajo o exigencias sean mayores, eso no importaría si cualquier profesor o actor escolar se sintiese apreciado y valorado.

D: «[...] yo considero que el liderazgo de una [...] de las personas deben de ser muy cercanos a su gente, tengo muy claro eso, muy cercano a la gente para que nos demos cuenta de cuáles son sus necesidades, sin embargo, yo creo que para 
eso existen las estructuras, para eso existen los organigramas, para eso existe la delegación de la autoridad, entonces yo pienso que mi estilo de liderazgo es de manera horizontal».

D: «[... ] yo no espero que la gente que está allá abajo, el docente, tenga que llegar directamente a mí para resolver un problema, para que pueda desarrollarse como docente, para trabajar como persona, para eso yo les doy a mis subdirectores y a mis jefes de departamento liderazgo, les doy capacidad para que tomen decisiones y puedan resolver los problemas desde allá abajo sin que tengan que venir a mí para que yo me pueda dedicar a otras cosas; digamos de planeación, administración, de toma de decisiones, porque si yo voy a resolver la situación de cada maestro, pues para la planeación, administración, toma de decisiones, no voy a hacer nada».

$\mathrm{D}:$ «Entonces yo creo que, que los docentes deben de decir que no estoy cercano a ellos, porque realmente no lo estoy, o sea, no voy a diario a sus oficinas, y no es porque no lo quiera hacer, sino porque no tengo tiempo».

D: «[... $]$ hay que hacerlos sentir importantes. No hay nada mejor que un empleado halagado».

D: «[... $]$ aunque muchos de ellos no sean afines a la dirección, hay que hacerlos sentir como elementos importantes en el centro».

$\mathrm{Al}$ parecer, el director entrevistado manifiesta que el humanismo es parte de su ser, y que le ha permitido obtener un trabajo más productivo de sus alternos. Respecto a ello, Blanchard y Bowles (2010), en su propuesta de mejora de organizaciones, plantean una filosofía sumamente integral que rescata el lado humano y eficiente del grupo de trabajo. Dentro de esta filosofía se propone el concepto de la ardilla que perfectamente ilustra la necesidad de que todo actor organizacional requiere contantemente que le sea expresado el valor de su trabajo para el centro escolar.

A manera de conclusión, en la figura 4 se muestran los elementos más representativos respecto de lo que los profesores esperarían de su director, de lo que debería caracterizar a todo líder educativo, y lo que un director considera poseer. 


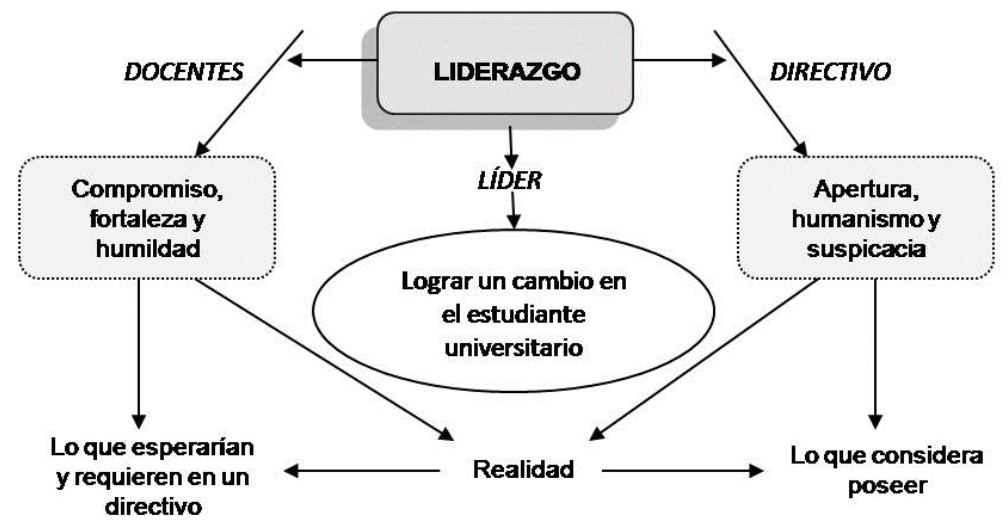

Figura 4. Perspectivas sobre el liderazgo, según el actor educativo.

Al llegar a esta parte del análisis, el lector tiene en sus manos información que le permite emitir un juicio respecto de lo que comentan estos actores de las instituciones estudiadas: profesores y directivos. Cada uno en su postura y, con base en las actividades y roles que les competen, reconocen lo que caracteriza su trabajo diario y lo que esperarían del alterno. Sin embargo, y como bien ilustra el gráfico anterior, ambos actores establecen sus perspectivas pero al tenerlas en comparación distan de lo que debería ser compartido.

Parece interesante cómo los profesores conciben a un líder educativo como aquel sujeto que logra un cambio en la vida de los estudiantes; este punto refleja el valor que podría tener un profesor en la educación universitaria. Al parecer, si un profesor alienta e involucra a los estudiantes en las actividades que este formador realice, es muy probable que el joven se vislumbre con un futuro prometedor, se interese en continuar activamente en su preparación, y lo convierta en un ciudadano activo, en búsqueda de mejores oportunidades.

Por otro lado, y sin parecer redundante con lo explicado en párrafos anteriores, es interesante notar lo que el directivo describe como sus adeptos de líder. Este personaje afirma trabajar con una constante apertura y humanismo, logrando con esto cercanía y confianza de su personal. De igual manera, aparece un elemento que el director traduce como habilidad de gran importancia para lograr sus propósitos, se habla de la suspicacia que todo directivo debe desarrollar para identificar a sus subalternos que pudieran contraponerse a esta autoridad.

De forma concreta parece que esta apertura y humanismo que comenta el director como cualidades que posee son cercanamente algunas características que los profesores desearían que su líder organizacional poseyese. $\mathrm{Y}$ aunque sea una ironía, cada actor tiene su propia postura e imagen construida, siendo lamentable que en la realidad aún falta para que esa similitud sea verdad. 


\section{Conclusiones}

Las personas que inciden con su conducta, actitudes y valores en la organización son las que definen y viven las políticas y lineamientos que norman la vida organizacional. Las personas también definen los procesos de trabajo, dividiéndolos en procedimientos, mismos que establecen el quehacer cotidiano de la institución (Zepeda, 1999). En las instituciones de educación superior (IES), existen agentes que ocupan altos mandos, lo cual les permite ejercer el poder con mayor facilidad. Ante la gran variedad de profesiones y responsables existentes en las IES, se considera necesario e importante conocer de manera específica qué y cómo hace cada uno de ellos la función que le corresponde, de tal manera que a partir del desempeño que tengan en sus cargos, la institución llegue a ser de calidad.

El liderazgo es un elemento de gran importancia en este estudio, y más relacionado al actor que encabeza el centro en cuestión. Los directivos conciben a un líder escolar como una persona con mucha apertura a críticas, que no le incomoda la opinión de los demás, y que permite recibir propuestas de mejora para la organización. Así como una persona humana, con actitud de servicio para los demás, olvidándose de su posición jerárquica. Ambas características son parte de la personalidad del directivo como parte de su ser y como estrategia para acceder a la atención de otros.

Vale la pena comentar que las características descritas por el director son parecidas a las que los profesores desean que pueda poseer su autoridad actual, lo que parece ser una total paradoja, cuando los escenarios son opuestos.

Por otro lado, el director cree que para ser un buen líder, que logre sus objetivos en beneficio de la organización, es necesario implementar ciertas técnicas que le faciliten ese camino, y como medida personal considera que tener suspicacia le permite identificar a aquellos actores que podrían ser un obstáculo para él. Esta suspicacia se desarrolla a través de una observación continua, de mirar por los pasillos, de identificar a aquellos que discuten durante las reuniones, así como de ver a los grupos formados en situaciones formales e informales. Todo ello le permite prever situaciones de conflicto según las actuaciones de los demás, conocer las coaliciones o alianzas, $y$ determinar a quiénes se debe dirigir para atender anticipadamente una situación.

En la educación superior mexicana, quienes logran ocupar un puesto o responsabilidad directiva generalmente son docentes que realizaron actividades en el pregrado o posgrado, y que a partir de una trayectoria en la institución y su vinculación son actores y grupos de decisión afines, son designados en puestos directivos o de gestión (Marúm, Curiel y Rosario, 2015). Esto lleva a pensar con mayor razón que el asunto del liderazgo en educación superior debe trabajarse 
mucho más, con la idea de establecer mejores parámetros de designación, evitando que asuntos de poder intervengan, y con la conciencia de que, como afirma Anderson (2010), «es fácil hablar de las prácticas claves del liderazgo directivo eficaz. No es tan fácil implementarlas» (p. 47). Y es así, pues cambiar implica llevar a cabo todo un proceso complejo.

\section{Bibliografía}

Aguilera, M. (2008). «La función directiva en secundarias públicas mexicanas: un acercamiento cualitativo desde la perspectiva de la eficacia escolar». Organizaciones educativas al servicio de la sociedad (vol. 2) (CD-ROM). Recuperado el 23 de octubre de 2014 de http://dialnet.unirioja.es/servlet/ articulo? codigo $=2873077$.

Anderson, S. (2010). «Liderazgo educativo: Claves para una mejor escuela». Psicoperspectivas, 9(2), 34-52.

Asociación Nacional de Universidades e Instituciones de Educación Superior (ANUIES) (2006). Estatuto de la Asociación Nacional de Universidades e Instituciones de Educación Superior de la República Mexicana A. C. México: Autor. Recuperado el 28 de febrero de 2014 de <http://www. anuies.mx/la_anuies/normatividad/estauto2006/estatuto_2006.pdf >.

Blanchard, K. y Bowles, S. (2010). ¡A la carga! (Gung Ho). Cómo aprovechar al máximo el potencial de las personas de su empresa. Colombia: Norma.

Blase, J. y Blase, J. (2002). «The micropolitical perspective». En: Blasé, J. (ed.). The Politics of life in schools: Power, conflict and cooperation. Newbury Park, CA: SAGE.

Bush, T. (2016). «School Leadership and Management in England: The Paradox of Simultaneous Centralisation and Decentralisation», Research in Educational Administration E Leadership, 1(1), 1-23.

Cáceres, M. ${ }^{a}$ P. (2007). Estudio del liderazgo estudiantil en la Universidad de Granada desde una perspectiva de género (Tesis doctoral con Mención Europea). Universidad de Granada, España.

Cantón, I. y Arias, A. (2008). «La dirección y el liderazgo: aceptación, conflicto y calidad». Revista de Educación, 345(enero-abril), 229-254. Recuperado el 8 de mayo de 2015 de <http://www.revistaeducacion.mec.es/re345/ re345_10.pdf>.

Denzin, N. (1989). The research act. 3. a ed. EUA: Prentice Hall.

Farías, L. y Montero, M. (2005). «De la transcripción y otros aspectos artesanales de la investigación cualitativa». International Journal of Qualitative 
methods, 4(1). Recuperado el 29 de noviembre de 2014 de <http:/ / www.ualberta.ca/iiqm/backissues/4_1/pdf/fariasmontero.pdf $>$.

Fernández, M.; Álvarez, M. y Herrero, E. (2002). La dirección escolar ante los retos del siglo XXI. España: Editorial Síntesis.

Flyvbjerg, B. (2001). Habermas y Foucault: pensadores de la sociedad civil. Estudios sociológicos. México, Colegio de México, núm. 19, 295-324.

Frost, D. (2010). «Teacher leadership and educational innovation». Zbornik Instituta za pedagoska istrazivanja, 42(2), 201-216.

Foucault, M. (1991). Microfísica del poder. Madrid: Las ediciones de la Piqueta. GAGO, F. (2008). «Liderazgo en la pecera (implicaciones para la dirección escolar)». Organizaciones educativas al servicio de la sociedad, vol. 2. (CD-ROM). Recuperado el 9 de octubre de 2015 de http://dialnet.unirioja.es/servlet/ articulo? codigo $=2872640$.

García, I. (2008)* «Liderazgo distribuidoः una teoría emergente aplicada al liderazgo educativo». Organizaciones educativas al servicio de la sociedad, vol. 2. (CD-ROM). Recuperado el 11 de marzo de 2014 de <http://dialnet.unirioja.es $/$ servlet $/$ articulo?codigo $=2872643>$.

González, J; Silva, P.; Antúnez, S. y Carnicero, P. (2008). Análisis de necesidades de formación de los directivos escolares en México. Organizaciones educativas al servicio de la sociedad, vol. 2. (CD-ROM). Recuperado el 4 de agosto de 2014 de <http://dialnet.unirioja.es/servlet/articulo?codigo=2873149>.

Johnson, J. y Uline, C. (2005). «Preparing Educational Leaders to Close Achievement Gaps». Theory into practice, 44(1) febrero, 45-52. Recuperado el 23 de marzo de 2013 de <http://pdfserve.informaworld. com/522439_770775852_789375037.pdf >.

Lorenzo, M. (2005). «El liderazgo en las organizaciones educativas: revisión y perspectivas actuales». Revista Española de Pedagogía, año LXIII (232), pp. 367-388. Recuperado el 26 de mayo de 2015 de <http://revistadepedagogia. org/content/view/57/44/lang,es/ >.

Lussier, R. y Achua, C. (2002). Liderazgo. Teoría, aplicación y desarrollo de habilidades. México: Internacional Thomson.

Martínez Rizo, F. (2000). Nueve retos para la educación superior. Funciones, actores y estructuras. México: Editorial ANUIES [En red]. Recuperado el 19 de agosto de 2015 de <http://books.google.com.mx/books?id=R6c->.

Martínez, S. (2008). «En qué consiste hoy un líder educativo». Organizaciones educativas al servicio de la sociedad, vol. 2. (CD-ROM). Recuperado el 12 de mayo de 2014 de <http://dialnet.unirioja.es/servlet/ articulo? codigo $=2872624>$. 
Marúm, E.; Curiel, F. y Rosario, V. (2015). Estudiantes frente al espejo. Percepciones de la calidad educativa en programas de licenciatura y posgrado en México. México: CUCEA UDG-CONACYT.

Maureira, O. (2004). «El liderazgo factor de eficacia escolar, hacia un modelo causal». REICE, Revista Electrónica Iberoamericana sobre Calidad, Eficacia y Cambio en la Educación, 2(001), enero-junio, 1-20. Recuperado el 18 de enero de 2015 de <http://dialnet.unirioja.es/servlet/articulo?codigo=904494>.

National Policy Board for Educational Administration (2015). Professional Standards for Educational Leaders 2015. Reston, VA: Autor.

Ruвio, B. (2006). Liderazgo en las organizaciones. Plan educacional para el desarrollo de futuros líderes (tomo 1). Colombia: ONIX.

Stake, R. (2010). Investigación con Estudios de Casos. 5a. Edición. España: Morata.

Tolentino, J. (2007). Gestión Académica de las Instituciones de Educación Superior. La formación de directivos. México: El Colegio de Veracruz.

Trujillo, J. (2007). «Análisis interno de la organización: liderazgo y micropolítica en un centro educativo». Innovación Educativa, 17, 37-52. Recuperado el 20 de julio de 2016 de <http://cat,inist.fr/?aModele=afficheN\&cpsi $\mathrm{dt}=18751279>$.

URIz, J. (1994). La subjetividad de la organización. El poder más allá de las estructuras. Madrid: Siglo XXI.

Zepeda, F. (1991). Psicología organizacional. México: Addison Wesley Longman. 